\title{
International symposium of junior neuroscience investigators (ISJNI19) UPM, 25-27 April, 2019 (Part-I)
}

Dehydroepiandrosterone sulfate (DHEAS) restores tyrosine hydroxylase (TH) immunoreactivity within the midbrain dopaminergic innervations in rat with mild hepatic encephalopathy

Omar EL Hiba ${ }^{1,2 *}$, Gamrani $\mathrm{H}^{1}$, Chatoui $\mathrm{H}^{1}$, Aitihya $\mathrm{M}^{1}$ and Ahboucha $S^{1}$

${ }^{1}$ Neuroscience, pharmacology and Environment, Cadi Ayaad University, Faculty of Science Semlalia

${ }^{2}$ Nutrition and Food Sciences laboratory, Faculty of Sciences, Chouaib Doukkali University, El Jadida, Morocco

Hepatic encephalopathy (HE) is a neuropsychiatric disorder occurring as a consequence of both acute and chronic liver failure. Advanced HE is generally accompanied with extrapyramidal symptoms including rigidity and tremor, which may reflect alterations of the dopaminergic system. Recently we reported a beneficial effect of the neuroactive steroid dehydroepiandrosterone sulfate (DHEAS) in cirrhotic rats, however the mechanisms of such an effect by DHEAS were not addressed. In the present study, we describe the changes of the dopaminergic system occurring in the cirrhotic rats and concomitantly we investigated the effect of DHEAS on this system in Sprague-Dawley rats using the expression of tyrosine hydroxylase (TH) as a neuronal marker. Rats were submitted to bile duct ligation (BDL) surgery and TH immunohistochemistry was assessed in the Substantia nigra pars compacta(SNc), striatum, ventral tegmental area (VTA) and the cortex. $\mathrm{TH}$ immunoreactivity showed a significant diminution in both SNc and VTA concomitantly with the cortical and the striatal outputs in the BDL rats vs. controls. Three daily injections of $5 \mathrm{mg} / \mathrm{kg}$ of DHEAS to BDL rats significantly normalized $\mathrm{TH}$ expression decrease in both SNc and VTA as well as dopaminergic projections to the striatum and the cortex of BDL rats. The present data support an involvement of the dopaminergic system in mild $\mathrm{HE}$ and a possible beneficial effect of the neurosteroid DHEAS as a potential pharmacological treatment of mild HE.

Key words: cirrhosis, tyrosine hydroxylase, substantianigra pars compacta, ventral tegmental area, dehydroepiandrosterone sulfate, rat

Neuropathology of hepatic encephalopathy: A focus on ammonia neurotoxicity

\section{Omar EL Hiba ${ }^{1,2}$}

${ }^{1}$ Neuroscience, Pharmacology and Environment Unit, Faculty of Sciences Semlalia, Cadi Ayyad University, Marrakech, Morocco

${ }^{2}$ Nutrition and Food Sciences laboratory and Nutritional Physiopathologies Team, Faculty of Sciences, Chouaib Doukkali University, El Jadida, Morocco
Hepatic encephalopathy (HE) is a complex neuopsychiatric disorder arising from both acute as well as chronic hepatic injuries. The first characterization of the HE syndrome was in the 1890's by the team of Pavlov and Nencki who assessed the physiological consequences of feeding with meet in portocaval shunted (PCS) dogs, which bypassed nitrogen-rich blood away from the liver. The authors observed a rapid deterioration of the neurological features of the dogs and finally hepatic coma. Later, ammonia was associated to those symptoms and has been considered as one of the key elements of HE neuropathology. At high concentration, ammonia elicits profound brain metabolic dysfunctions and astrocytic morphological changes called "Alzheimer type II astrocytosis" with altered mitochondrial functions. Otherwise, experimental in vivo and in vitro studies have revealed a possible direct impact on the neuronal function via interference with excitatory and inhibitory neuronal systems which may be considered as key elements in the pathogenesis HE.

Key words: hepatic encephalopathy, ammonia, Alzheimer type II astrocytosis

\section{A comparison of astroglia reactivity in the brain of a rat model of mild hepatic encephalopathy}

Omar EL Hiba ${ }^{1,2^{*}}$, Gamrani $\mathrm{H}^{1}$, Chatoui $\mathrm{H}^{1}$, Aitihya $\mathrm{M}^{1}$ and Ahboucha $S^{1}$

${ }^{1}$ Neuroscience, pharmacology and Environment, Cadi Ayaad University, Faculty of Science Semlalia

${ }^{2}$ Nutrition and Food Sciences laboratory, Faculty of Sciences, ChouaibDoukkali University, El Jadida, Morocco

Hepatic encephalopathy (HE) is a neuropsychiatric disorder that occurs in both acute and chronic liver failure. However, the pathomechanisms of the disease remains obscure. Neuropathological studies have demonstrated a primary gliopathy in humans as well as in animal models of chronic and acute liver failure. Here, we have investigated in an animal model of mild HE: the bile duct ligated rat (BDL) at the cirrhotic stage (4 weeks after surgery), the expression of the key marker of mature astrocytes, the glial fibrillary acidic protein (GFAP) in different brain areas such as: Substantia nigra pars compacta (SNc), Ventral tegmental area (VTA), hippocampus, dorsal striatum and brain cortex by means of immunohistochemistry. The immunohistochemical study showed, in BDL compared to the operated controls (shams), a diminished astrocyte reactivity corresponding to a loss of GFAP expression within SNc, VTA, hippocampus and dorsal

${ }^{\star}$ Correspondence to: Omar EL Hiba, Department of Biology, Chouaib Doukkali University, Route Ben Maachou, 24000, El Jaddia, Morocco, E-mail: omar. elhiba@edu.uca.ac.ma

Received: April 19, 2019; Accepted: April 26, 2019; Published: April 30, 2019 
striatum $(\mathrm{p}<0.05)$, whereas in the brain cortex astrocytes appeared strongly immunoreactive with increased GFAP expression $(\mathrm{p}<$ $0.05)$ as compared to shams. Our finding demonstrated differential astroglialresponses which depend to the specificity of the area investigated and its particular neuronal neighboring environment, and could have possible outcomes on the diverse neuronal functions especially those observed during the different episodes of hepatic encephalopathy.

Key words: mild hepatic encephalopathy, BDL, GFAP, astrocyte, substantia nigra pars compacta, ventral tegmental area, dorsal striatum, hippocampus, immunohistochemistry

The response of the subcommissural organ secretory activity in rat with bile duct ligation induced mild hepatic encephalopathy

\section{Omar EL Hiba ${ }^{1,2^{*}}$, Gamrani $\mathrm{H}^{1}$ and Ahboucha $\mathrm{S}^{1}$}

${ }^{1}$ Neuroscience, pharmacology and Environment, Cadi Ayaad University, Faculty of Science Semlalia

${ }^{2}$ Nutrition and Food Sciences laboratory, Faculty of Sciences, ChouaibDoukkali University, El Jadida, Morocco

Hepatic encephalopathy is a common neuropsychiatric complication of acute and chronic liver failure. Whether brain structures with strategic positions in the interface of blood-brain barriers such as the circumventricular organs are involved in hepatic encephalopathy is not yet established. Among the circumventricular organs, the subcommissural organ secretes a glycoproteinknown as Reissner's fiber, which condenses and forms an ever-growing thread-like structure into the cerebrospinal fluid. In the present work we describe the Reissner's fiber material within the subcommissural organ and its serotoninergic innervation in an animal model of chronic hepatic encephalopathy following bile duct ligation in experimental rats. The study involved immunohistochemical techniques with antibodies against Reissner's fiber and 5-hydroxytryptamine (5-HT). Four weeks after surgical bile ductligation, a significant rise of Reissner's fiber immunoreactivity was observed in all subcommissural organ areas compared with controls. Moreover, significant Reissner's fiber immunoreactive materials within the ependymaand inside the parenchyma close to the ventricular borders were also seen in bile duct ligated rats, but not in control rats. Increased Reissner's fiber material in bile duct ligated rats seems to be related to a reduction of 5-HT innervation of the subcommissural organ, the ventricular borders and the nucleus of origin, the dorsal raphe nucleus. Our data describe alterations of the subcommissural organ/Reissner's fiber material and the subcommissural organ 5-HT innervation probably due to a general 5-HT deficit in bile duct ligated rats.

Key words: subcommissural organ, serotonin, reissner's fiber, hepatic encephalopathy, immunohistochemistry, rat

\section{Effect of acute exposure to manganese chloride on pain perception}

\section{Anas Bourziq ${ }^{1 \star}$, Radouane $\mathrm{EL}^{1}$, Far $\mathrm{I}^{1}$ and Gamrani $\mathrm{H}^{1}$}

${ }^{1}$ Cadi Ayyad University, Faculty of sciences Semlalia, Neurosciences, Pharmacology and Environment Unit, Marrakesh, Morocco

Manganese, an essential heavy metal for human body, it plays a key role in many biological functions. Indeed, it acts as a cofactor for many metalloenzymes, especially in the central nervous system.
The paramagnectic properties of Mn allow its use as a contrast agent for magnetic resonance imaging (MRI). Therefore, Mn enhanced magnetic resonance imaging (Mn-MRI) provides valuable information about central nervous system functions, and MEMRI is largely used in functional exploration of pain perception. Data on the effect of manganese on the perception of pain are rare and poorly described. However, in case of Mn deficiency, women develop an increase in pain during the premenstrual phase of the ovarian cycle. Thus, the aim of our study is to demonstrate the effect of acute exposure to $\mathrm{Mn}$ on the perception of pain in mice. We used sub-acute Mn intoxication model, and we assessed pain perception in animal using hot/cold plate. Indeed, we found $\mathrm{Mn}$ to decrease in pain sensation among intoxicated animal when compared to controls. Finally, these results indicate that acute exposure to manganese altered the perception of pain which could limit the use of the powerful imaging tool MEMRI in pain exploration.

Key words: manganese, exposure, pain perception

Oxidative stress induced by lead in Meriones shawi: A possible link with neurobehavioral alterations

Oukhrib Mjid*, Tamegart Lahcen Bouyatas, My Mustapha and Gamrani Halima

\section{Faculty of Sciences Semlalia, Cadi Ayyad University}

Lead is not known to have any physiological role in the body but is ubiquitous in nature. In our country there are many sources of lead exposure, in particular paints and recycling of batteries. Lead is a persistent metal and is present in water, soil and dust. After being absorbed through GIT or respiratory system, it is stored in soft tissues as well as bones. Lead tends to affect many organ systems in the body particularly the nervous system. Studies have shown that $\mathrm{Pb}$-exposure could be a risk factor in the development of Parkinson's disease(PD).

The latter is related to dopaminergic deficiency that may be triggered by genetic and environmental factors suchas $\mathrm{Pb}$ intoxication. In this study, we have evaluated the neurotoxic effect of $\mathrm{Pb}(3 \mathrm{~g} / \mathrm{l})$ by oral gavage on locomotor performance in Merione shawi. Our study will focus on the negative effects of oxidative stress on the brain of meriones shawi, since it is the organ most exposed to the oxidation due to the high phospholipid content of neuronal membranes and the link existing with the development of neurodegenerative pathologies such as $\mathrm{PD}$. The locomotor activity dysfunction in $\mathrm{Pb}$-intoxicaed meriones is linked to the possible effects of lead on the nigro-striatal pathway. In this study, we have revealed a new neurotoxic effect of lead and could induce neurodegenerative disorders, especially, which implying the nigro-striatal pathway injuries, like PD, trigged by heavy metals.

Key words: nigro-striatal pathway, lead neurotoxicity, locomotion, meriones shawi,s oxidative stress

\section{Neurotoxicity of manganese: A focus on neurodevelop- mental aspects}

Hafida EL Ghachi, Radouane EL fari, Abdellatif Abbaoui and Halima Gamrani

Cadi Ayyad University, Faculty of sciences Semlalia, Neurosciences, Pharmacology and Environment Unit, Marrakesh, Morocco

The establishment of a complex and powerful brain able to ensure vital functions of the organism requires a succession of processes highly exquisite delicately coordinated in time. The least disruption of any step of development can heavy affect the ultimate functions. 
The development of central nervous system (CNS) exhibits some periods of vulnerability, in which particular toxicants at low levels, even harmless in adults may cause significant detrimental effects. These vulnerable life stages of development occur in utero, during infancy and early childhood. During the pregnancy, and especially during neuronal differentiation process, this vulnerability to non-physiological levels of any compound is increased, highlighting thus, the importance of environmental care.

Manganese (Mn), a heavy metal, despite its essentiality and its role in many enzymatic systems it is considered as a neurotoxic element at higher levels of exposure because of its wide use in different domains (metallurgical industry, agronomy...). High levels of Mn may damage the basal ganglia leading to a syndrome analogous to Parkinson's disease with motor and cognitive impairments that include dystonia, bradykinesia and rigidity.

Exposure to manganese that naturally occurs in the environment such as in underground water used for consumption, in children, particularly vulnerable to environment toxicants, showed symptoms including cognitive deficits, behavioural damages, decreased both intellectual quotient and school performance.

The neurotoxic effects of $\mathrm{Mn}$ were first identified in adults following occupational exposure to high concentrations. However, studies linking manganese exposure with the development of the nervous system are still unsatisfying. Hence, we used prenatal Mn intoxication model to assess $\mathrm{Mn}$ effects on pups development and cognitive performances.

Key words: manganese, neurotoxicity, neurodevelopmental

\section{Altered working memory and hippocampal astroglia morphology in rat with reserpine-inducedprogressive Pakinson's disease}

Ahmed Draoui ${ }^{1,2}$, Omar EL Hiba ${ }^{1,2}$, Abdelaati EL Khiat ${ }^{1}$, Radouane EL Fari ${ }^{1}$ and Halima Gamrani ${ }^{1}$

${ }^{1}$ Neuroscience, Pharmacology and Environment Unit, faculty of Sciences Semlalia, Cadi Ayyad University, Avenue My Abdellah, B.P. 2390, Marrakesh, Morocco

${ }^{2}$ Nutrition and Food Sciences laboratory, faculty of Sciences, ChouaibDoukkali University El Jadida, Route Ben Maachou, B.P. 20, Avenue des Facultés, El Jadida, Morocco

The aim of this study is to assess the evolution of the working memory at different stages of the prodromal phase of a rat model of $\mathrm{PD}$, using repetitive reserpine administration at low dose. The study was carried out in rat with repeated i.p reserpine administration. Working memory was assessed by the Novel Object Recognition test (NOR) and the T-maze at to stages of prodromal phase of the disease. By means of immunohistochemistry, the morphological changes of astroglia within hippocampus (using anti-GFAP marker) was exanimated at the latest stage of the disease. Our data show the deterioration of working memory, which was accompanied by a striking change in both density and morphology of the astrocyte at the level of the hippocampus. The present study has brought evidence of an early deficit of working memory rather the latest stage of the prodromal phase of PD. Such deficit could arise from the loss of the astroglialmorpho-functional changes within the hippocampus leading to possible neurophysiological disturbances of the different neighboring neuronal populations involved in short working memory.

Key words: Parkinson's disease, reserpine, working memory, hippocampus, astroglia

Copyright: (92019 Hiba OEL. This is an open-access article distributed under the terms of the Creative Commons Attribution License, which permits unrestricted use, distribution, and reproduction in any medium, provided the original author and source are credited. 